1 Longitudinal cell division is associated with single mutations in the FtsZ-recruiting SsgB in Streptomyces

2

3 Xiansha Xiao ${ }^{a, \#,}$, Joost Willemse ${ }^{a, \#, ~ P a t r i c k ~ V o s k a m p ~}{ }^{b}$, Xinmeng Li $^{\mathrm{c}}$, Meindert Lamers ${ }^{\mathrm{d}}$, Jan Pieter

$4 \quad$ Abrahams $^{\mathrm{e},},{ }^{*}$ Navraj Pannu ${ }^{\mathrm{b}}$, Gilles P. van Wezel ${ }^{\mathrm{a},{ }^{*}}$

5

6 Molecular Biotechnology, Leiden University, PO Box 9505, 2300RA Leiden, The Netherlands

7 b Biophysical Structural Chemistry, Leiden University, PO Box 9502, 2300RA Leiden, The Netherlands

$8{ }^{c}$ LIC/Energy \& Sustainability, Leiden University, PO Box 9502, 2300RA Leiden, The Netherlands

$9{ }^{\mathrm{d}}$ Leiden University Medical Center, P.O. Box 9600, 2300RC Leiden, The Netherlands

10 e Paul Scherrer Institute, Bio-nano diffraction Biozentrum/ C-CINA, Basel University, CH-5232, Villigen PSI,

\title{
11 Switzerland
}

12

13 \# these authors contributed equally to the work

14 * Corresponding authors. J.P. Abrahams: Tel. +41 563104612, Email: Jan-Pieter.Abrahams@psi.ch; G.P. van

15 Wezel: Tel +31 715274310, Email: g.wezel@biology.leidenuniv.nl. 


\section{ABSTRACT}

18 In most bacteria, cell division begins with the polymerization of the GTPase FtsZ at the mid-cell, which

19 recruits the division machinery to initiate cell constriction. In the filamentous bacterium Streptomyces, cell

20 division is positively controlled by SsgB, which recruits FtsZ to the future septum sites and promotes Z-ring

21 formation. Here we show via site-saturated mutagenesis that various amino acid substitutions in the highly

22 conserved SsgB protein result in the production of ectopically placed septa, that sever spores diagonally

23 or along the long axis, perpendicular to the division plane. Ectopic septa were especially prominent when

24 cells expressed SsgB variants with substitutions in residue E120. Biochemical analysis of SsgB variant

25 E120G revealed that its interaction with - and polymerization of - FtsZ had been maintained. The crystal

26 structure of S. coelicolor SsgB was resolved and the position of residue E120 suggests its requirement for

27 maintaining the proper angle of helix $\alpha 3$, thus providing a likely explanation for the aberrant septa formed

28 in SsgB E120 substitution mutants. Taken together, our work presents the first example of longitudinal

29 division in a free living bacterium, which is explained entirely by changes in the FtsZ-recruiting protein

30 SsgB.

31 


\section{INTRODUCTION}

33 Bacterial cell division is mediated via the formation of a contractile ring that consists of the tubulin

34 homologue FtsZ. FtsZ polymerizes into a ring-like structure (the Z-ring) which serves as a scaffold for the

35 recruitment of other members of the cell division machinery or divisome (Adams and Errington, 2009).

36 The GTP-dependent polymerization of FtsZ is essential for the constrictive force. Cryo-electron microscopy

37 and cryo-electron tomography data suggest that the Z-ring consists of small discontinuous and single-

38 layered filaments form a continuous ring through lateral association (Szwedziak et al., 2014). By filament

39 sliding and a hinge-opening conformational switch, the Z-ring drives constriction of the cell membrane, as

40 shown by in vitro reconstitution and structural studies (Li et al., 2013; Szwedziak et al., 2014). Septum-site

41 localization in unicellular bacteria depends on FtsA and ZipA that anchor FtsZ polymers to the cell

42 membrane, with ZapA stabilizing the FtsZ filaments and promoting lateral interactions (Hale and deBoer,

43 1997; Pichoff and Lutkenhaus, 2002, 2005). In Escherichia coli, control of Z-ring timing and localization is

44 governed by the Min system and by nucleoid occlusion, which negatively regulate FtsZ polymerization

45 (Adams and Errington, 2009; Lutkenhaus, 2007; Margolin, 2005; Shapiro et al., 2009).

46 Due to its central role in cell division, FtsZ is essential in nearly all bacteria. Two exceptions are the

47 parasite Mycoplasma (Lluch-Senar et al., 2010), which has a reduced genome size and no cell walls, and

48 Streptomyces (McCormick et al., 1994; McCormick, 2009). Streptomycetes are filamentous Gram-positive

49 bacteria in the phylum of Actinobacteria, that have a complex mycelial life style (Barka et al., 2016). These

50 bacteria produce over $60 \%$ of all known antibiotics and many other bioactive natural products (Hopwood,

51 2007; van der Heul et al., 2018). Streptomycetes are model organisms for the study of multicellularity and

52 bacterial morphogenesis (Claessen et al., 2014; Flärdh and Buttner, 2009). Exponential growth of the

53 multi-nucleoid vegetative hyphae is achieved by apical growth and branching. At this stage of the life cycle,

54 cell division does not affect physical separation of the cells, but instead long syncytial cells are formed that

55 are separated by cross-walls (Wildermuth and Hopwood, 1970). When the developmental programme is 
switched on, streptomycetes produce aerial hyphae, that ultimate differentiate into chains of unigenomic

57 spores. Recent studies highlight that local membrane synthesis and branching may be an important divisome-independent mechanism for cell proliferation in Streptomyces (Celler et al., 2016; Yagüe et al., 2016).

Streptomycetes lack the canonical cell-division regulation systems such as Min and Noc (Jakimowicz and van Wezel, 2012). Instead, a positive control system has evolved, whereby FtsZ is actively recruited to the septum sites by SsgB, in concert with its paralogue SsgA (Willemse et al., 2011). SsgA and SsgB belong to the SsgA-like proteins (SALPs), a family of regulatory proteins that is unique in sporulating actinobacteria, of which SsgA and SsgB are required for sporulation-specific cell division in Streptomyces (Noens et al., 2005; Traag and van Wezel, 2008). Actinobacteria that form single spores, such as Micromonospora or Thermobifida, only have one SALP, namely the FtsZ-recruiting protein SsgB, while up to 14 SALPs can be found in those genera which form chains of spores, such as Streptomyces (van Dissel et al., 2014). In the early stage of division, SsgA orchestrates division by facilitating the correct localization of SsgB. With the help of the transmembrane protein SepG, SsgB directly recruits FtsZ to the future septum sites and tethers the Z-ring to the inner membrane (Willemse et al., 2011; Zhang et al., 2016). SsgB is the archetypical SALP, with a conserved function in the development of actinomycetes (Xu

72 et al., 2009). While the SsgB protein sequence varies strongly between less related Actinobacteria, the 73 protein is extremely well conserved within a genus, with a maximum of one amino acid variation, a feature 74 that has been applied for the phylogenetic analysis of closely related Actinobacteria (Girard et al., 2013).

75 In this work, we investigated the importance of individual residues in the localization of the septum during 76 sporulation-specific division, by creating a library of SsgB mutants and studying their effect on cell division 77 and morphogenesis. Single aa changes in SsgB had major effects on cell division, spore-wall synthesis, and DNA condensation and/or segregation. Remarkably, specific mutations led to the formation of additional septa with $10^{\circ}$ to $90^{\circ}$ rotation of the division plane. Such longitudinal fission has so far only been seen in 
80 the worm-associated bacteria Candidatus Thiosymbion oneisti and Thiosymbion hypermnestrae. In these

81 two bacteria, cell growth and longitudinal division are polarized by their symbiotic nematode hosts (Pende

82 et al., 2018). X-ray crystallography revealed major structural differences between the SsgB from S.

83 coelicolor and its distant orthologue from T. fusca. Our data support the predominant role of SsgB in the

84 accurate positioning of the division site and the placement of the Z-ring. 


\section{RESULTS}

\section{Mutational analysis of SsgB}

87 SsgB shows unusual conservation, with near complete conservation within a genus, and high divergence

88 even between related actinobacterial genera. To investigate this further, we analyzed the effect of point

89 mutations in SsgB on cell division and morphogenesis, using S. coelicolor as the model system. For this, we

90 first created a random mutant library using error-prone PCR, similar to the approach used previously for

91 the mutational analysis of SsgA (Traag et al., 2007). Mutant genes, preceded by (and transcribed from) the

92 original $\operatorname{ssg} B$ promoter region, were cloned into the low-copy number vector pHJL401 and introduced into

93 the $s s g B$ null mutant, followed by scrutiny of sporulation and cell division. To ascertain that the observed

94 phenotypes were not due to differences in SsgB expression, the mutant was also complemented with a

95 clone expressing wild-type SsgB, which gave a wild-type sporulation phenotype (see below). Additionally,

96 Western analysis was performed using anti-SsgB antibodies. Samples were equalized for protein content

97 and corrected based on the levels of elongation factor EF-Tu1 (Vijgenboom et al., 1994). This revealed an

98 average expression level of $77 \%+/-10 \%$ of the wild-type level.

99 Spores of S. coelicolor are grey-pigmented due to the production of the WhiE spore pigment

100 (Kelemen et al., 1998), while colonies developing non-sporogenic aerial hyphae are white; intermediate

101 phenotypes (reduced sporulation results in a light-grey pigmentation) also occur. This feature was utilized

102 to subcategorize all transformants into three groups: white, light grey and grey. The mean grey level of

103 growing patches was analyzed based on the scanner images. By this approach, the degree of sporulation

104 could be readily monitored (Figure S1 and Table S1). 232 clones were isolated from the transformants and

105 sequenced. Of these, 84 had no or silent mutations, 39 had multiple mutations and 65 had insertions or

106 deletions. Of the 42 remaining clones, 35 unique single substitutions were identified and these were

107 analyzed further. Out of 35 SsgB variants, six failed to sporulate and the others showed significant

108 sporulation defects or reduced sporulation (Figure S2, Table S1). 
To obtain more detailed insights into the morphological changes correlating to the substitution

110 mutants, the transformants expressing SsgB variants were subjected to transmission electron microscopy

111 (TEM) (Figure 1). Wild-type spores were homogeneous in size, with a thick electron-dense spore wall and

112 condensed DNA in the centre of the spores. Conversely, spores from transformants expressing SsgB

113 variants generally showed high variation in spore-wall thickness, spore size and shape, and frequently also

114 aberrant DNA segregation and/or condensation (Figure 1). Much to our surprise, in some cases up to $90^{\circ}$

115 rotation of the septal plane was seen, dividing the spores parallel to the growth direction of the hyphae.

116 This suggests that mutation of single SsgB residues may affect the coordination of cell division in aerial

117 hyphae of Streptomyces, as detailed below.

119 Rotation of the division plane due to single amino acid substitutions in SsgB

120 Based on the outcome of the random mutant library, 22 residues were selected for site-saturated

121 mutagenesis, namely W51, L88, A95, L96, L97 and the C-terminal residues 115-131 that are centered

122 around E120 that correlated to the surprising longitudinal division (Table S2). Each of these residues were

123 changed into on average 14 different amino acid residues using DNA synthesis (Table S2). Mutants for the

124 hydrophobic residues W51, L88, A95, L97, V115, P116 and P117, frequently had non-sporulating

125 phenotypes (Table S2). Variable spore sizes were seen in most of the mutants, with some also showing

126 irregular cell wall thickening (Figure 1). Importantly, thirteen mutants wherein E120 was replaced by either

$127 \mathrm{~A}, \mathrm{C}, \mathrm{F}, \mathrm{G}, \mathrm{H}, \mathrm{I}, \mathrm{K}, \mathrm{L}, \mathrm{N}, \mathrm{P}, \mathrm{Q}, \mathrm{S}$ or T produced septa with significant rotation of the division plane - in addition

128 to canonical septa. The angles of these ectopically positioned septa ranged from diagonal to longitudinal

129 (i.e., $90^{\circ}$ rotation, with septa parallel to the hyphal wall), of which $5.2 \%$ were positioned diagonally (529

130 out of 10257 ), and $0.8 \%$ longitudinally ( 86 of the 10257). See Figure 1B and Table S3. In addition to

131 mutants expressing SsgB E120 variants, longitudinal division was also seen in mutants expressing SsgB

132 variants V115G, G118V, L96I, L96P and L96S, whereby the latter three produced relatively few ectopic 
133 septa (Table S4). To the best of our knowledge, this is the first report of longitudinal cell division in any

134 free-living bacterium. To ascertain that longitudinal division does not occur in the wild-type strain under

135 the chosen conditions, over 1000 samples of the wild-type strain were checked by SEM and TEM, and not

136 a single rotated septum was observed.

137 In order to see if the longitudinal septation also resulted in physical separation of the severed

138 spores, we made impression prints of the strain expressing SsgB variant E120G, that had been grown for

1397 days on SFM agar plates. These spores were then fixed with $1.5 \%$ glutaraldehyde in PBS, followed by

140 dehydration using a graded series of acetone (70-100\%). This experiment clearly demonstrated that the

141 strain expressing SsgB E120G produces spores that are longitudinally sectioned in two and that this

142 process is completed by spore fission (Figure 2A, panels e-f). The fixation procedure led to some drying

143 artifacts in wild-type (Fig. 2A, panel a) and ssgB E120G (Fig. 2A, panels c-d) spores, but this effect was

144 clearly different from the longitudinal division.

145 Viability of the spores of mutants in which the residue E120 had been replaced by other amino

146 acids were compared to those of wild-type spores. For this, impression prints were stained with Syto9 for

147 viable spores and propidium iodide (PI) for dead spore, and imaged via fluorescence microscopy. While

148 wild-type SsgB spores were nearly all viable, those obtained from E120 substitution mutants varied a lot,

149 with 5-70\% dead spores, depending on the mutant (Figure S3). Like in de SEM experiments, longitudinal

150 septation could also be seen from the outside using light microscopy, indicative of unique cell fission

151 parallel to the hyphal wall (Figure 2B, panels a-c).

153 Localization and dynamics of SsgB variants

154 To confirm that longitudinal division in the aerial hyphae correlated to the localization of SsgB, chimeric

155 SsgB-eGFP and SsgB-G118V-eGFP fusions were created as described (Willemse et al., 2011). While wild-

156 type SsgB (Figure S4A, panel a) showed the typical pattern of foci on either side of the hyphal wall, the 
158 in both canonical septal rings (perpendicular to the hyphal wall) and with a certain frequency also septa 159 that were tilted by $90^{\circ}$ (marked by arrowheads in Figure S4A, panel c).

161 (Figure 3). The plotted graph of wild-type SsgB indicates its localization on either side of the hyphae wall.

162 Whereas, $\triangle \operatorname{ssg} B:: s s g B(G 118 V)$ and $\Delta s s g B:: s s g B(E 120 G)$ showed aberrant localization, which occasionally

163 resulted in longitudinal septation, where SsgB localized in the middle of the hyphae, consistent with the

164 observed longitudinal cell division. To gain insights into the dynamic association/dissociation of SsgB with

165 the divisome, monomeric exchange was examined via Fluorescence Recovery After Photobleaching (FRAP).

166 The recovery time after photobleaching was determined both on pre-septation foci as well as on septa.

167 No difference in dynamics was seen between wild-type SsgB and its G118V variant. Both showed a

168 recovery time of around $60 \mathrm{~s}$ (Figure S4B), which is similar to previously reported data (Willemse et al., 169 2011).

DNA content of over 500 spores of wild-type strain and $\triangle \operatorname{ssg} B:: s s g B(E 120 G)$ was studied. While

171 wild-type spores showed a normal DNA distribution, $\triangle s s g B:: s s g B(E 120 G)$ showed major variation in DNA

172 content (Figure S5A). As an illustration, one spore chain containing longitudinal divisions is shown with the

173 respective DNA content in each spore, which revealed 0.4 to 3.0 chromosomes for each spore in

$174 \Delta s s g B:: s s g B(E 120 G)$ (Figure S5B). Conversely, 0.83 to 1.15 chromosomes were observed in the wild-type

175 strains for each spore (Figure S5C).

\section{SsgB-E120 mutants assist in the polymerization of FtsZ filaments}

178 To establish whether SsgB E120G and E120A had retained the ability to interact with FtsZ, wt SsgB, SsgB

179 variants E120A and E120G, as well as a C-terminally truncated version of SsgB (SsgB $\Delta$ C, spanning 1-114 aa)

180 were expressed and purified and then tested using a pelleting assay. SsgB of S. coelicolor (ScSsgB) was 
181 enriched in the pellet after incubation with FtsZ under polymerizing conditions (i.e. in the presence of GTP

182 and $\mathrm{Mg}^{2+}$ ), while about $50 \%$ of FtsZ was recovered by centrifugation (Figure 4A). ScSsgB variants E120A,

183 E120G and SsgB $\Delta$ C all pelleted in the presence of FtsZ when GTP and $\mathrm{Mg}^{2+}$ were added (Figure 4A),

184 although less efficiently as compared to wt ScSsgB. Neither FtsZ nor SsgB was recovered by centrifugation

185 in the absence of GTP (Figure 4A), confirming that they did not form aggregates.

187 alone formed short, straight and single-stranded filaments in the presence of GTP (Figure 4B, panel a).

188 Addition of wt SsgB promoted the formation of bundled filaments (Figure 4B, panels b and c), similarly as

189 seen for SsgB from T. fusca (Willemse et al., 2011). Extended and bundled filaments were also observed in

190 the presence of SsgB E120A, E120G and SsgBAC, with E120A showing fewer and more 'loose' bundles

191 (Figure 4B, panels d, e and f). Taken together, these data show that mutation of E120 or by deletion of the

19223 C-terminal residues does not prevent the binding of ScSsgB to FtsZ, whereby the mutant proteins still

193 promote the formation of FtsZ filaments.

\section{Crystal structure of S. coelicolor SsgB}

196 In order to gain more insights into the structure-function relationship for key SsgB residues, the structure

197 of ScSsgB was resolved via X-ray crystallography. For this, hexahedron crystals were obtained, and based

198 on this, a homo-trimer was resolved at $2.1 \AA$ (PDB ID Code 6SLC) with eight molecules per asymmetric unit

199 (Table 1). The 13 aa residues at the C-terminus were highly mobile and could therefore not be modeled,

200 due to lack of electron density. Each subunit was arranged as an $\alpha+\beta$ fold, with seven $\beta$-strands packed

201 into a barrel structure, covered by three $\alpha$-helices (Figure 5A), which strongly resemble those of TfSsgB

202 structure(Xu et al., 2009) (Figure 5C). The root-mean-square deviation (r.m.s.d.) is $1.9 \AA$ with $92 \%$ of all

203 residues aligned in ScSsgB and 87\% in TfSsgB (aa sequence identity between these two proteins is 46\%).

204 A superimposition of ScSsgB and TfSsgB subunits is shown in Figure 5E. ScSsgB trimer adopts a "whirly" 
shape and is assembled through an antiparallel $\beta$-sheet interaction between $\beta 1$ from one subunit and $\beta 4$ through $\alpha$-helices (Figure 5D). The ScSsgB trimer forms a 12-stranded beta-barrel with an inner diameter of about $20-25 \AA ̊$ (Figure 5B).

\section{Analysis of single mutations and mapping of key residues}

211 Key residues that correlated to the occurrence of longitudinal cell division were mapped onto the ScSsgB

212 trimer structure (Figure 6A, Figure S7A). Mutations that correlate to residues that are evolutionary

213 conserved in all SALPs are underlined (Figure 6A, Figure S7B). Residues V115, G118 and E120 cluster

214 together, and are centered on the lid of the beta-barrel consisting of $\alpha 1, \alpha 2-\alpha 3$ and $\beta 1-\beta 2$ loop, which is

215 close to the interface between $\alpha 3$ and the rest of the subunit (Figure 6B). Consistent with their strategic

216 localization in the $\alpha 2-\alpha 3$ loop, amino acid substitutions V115G, G118V or E120G resulted in the formation

217 of aberrant tilted septa in addition to the canonical septa perpendicular to the hyphal wall, with some

218 septa showing full $90^{\circ}$ rotation of the division plane. Residue E120 plays a key role in maintaining the

219 proper angle between $\alpha 3$ and the rest of the protein. Three hydrogen bonds are formed between the E120

220 side chain and the main chains of E120, T119 and G118 in the $\alpha 2-\alpha 3$ loop region. Besides, E120 and V115

221 provide two additional salt bridges to R55. Interestingly, a $\pi-\pi$ interaction and a hydrogen bond were

222 observed for E120-Y35 and H121-Y35. All these interactions stabilize the angle of $\alpha 3$ (Figure 6C),

223 supporting the importance of the proper angle between $\alpha 3$ and the rest of the protein to the function of

224 ScSsgB.

226 Molecular dynamic simulation of SsgB E120G

227 Our work demonstrated that residue E120 plays a key role in maintaining the proper angle of $\alpha 3$ relative 228 to the rest of the protein. Mutation of this residue would disrupt the interaction, and changing the angle 
of $\alpha 3$ may drive rotation of the septum plane, and thus explain the observed longitudinal cell division. The

230 heading and the high B-factor of the existing residues in the $\alpha 3$-helix (Figure S8) suggests that it can extend

231 flexibly to the center of the trimer and serve as a lid for the mentioned beta-barrel at the center of the

232 structure. Despite many attempts under different crystallization conditions, we failed to obtain crystals for

233 SsgB E120G or SsgB E120A. In silico molecular dynamics revealed that while the $\alpha 3$ helix of the wild-type

234 SsgB protein is not affected significantly by the simulations, with a 2.7 and $3.0 \AA$ of distance respectively

235 between E120 and R55 (Figure 7A), the $\alpha 3$ helix of SsgB E120G flips some $90^{\circ}$ outwards of the tight trimer,

236 with the distance increasing to 10.6 and $10.9 \AA$, respectively (Figure 7B). This provides supportive evidence

237 that the angle of the $\alpha 3$ helix may indeed play a key role in determining the orientation of the septum

238 plane.

240 Oligomerization Studies of SsgB

241 Crystallographic data obtained for SsgB from T. fusca (Xu et al., 2009) and for S. coelicolor (this work)

242 suggested that SsgB forms trimers. To ascertain this, size-exclusion chromatography (SEC) of wt ScSsgB and

$243 S c S s g B \Delta C$ was conducted. SEC results indicated that freshly purified wt ScSsgB and $S c S s g B \Delta C$ mainly

244 existed as a monomer in solution (Figure S9A), while the SEC experiment of the same batch of protein

245 showed the existence of a trimer after a short-time storage in $-80^{\circ} \mathrm{C}$, revealing the conversion of monomers

246 to trimers over time (Figure S9B).

\section{DISCUSSION}

250 SALPs play a central role in controlling the steps of sporulation-specific cell division in Streptomyces.

251 Inspired by the extremely high conservation of the SsgB protein in Streptomyces species, with natural

252 variants only found in aa $128(\mathrm{Q}, \mathrm{R}$ or $\mathrm{T})$, we studied the effect of point mutations in the protein on cell 
division and morphogenesis of the model strain S. coelicolor. As expected, many mutants showed

254 morphological defects relating to cell division and sporulation, including varying spore sizes, aberrant DNA

255 segregation and condensation, and cell wall thickening. Surprisingly, SsgB substitutions L96P, V115G,

256 G118V and various changes in E120 caused the formation of additional septa that section the spores

257 diagonally or longitudinally, perpendicular to the canonical septa. This is the first example of longitudinal

258 division in a free-living bacterium. Diagonal and longitudinal Z-rings and septa always coincided with

259 canonically oriented Z-rings/septa. Furthermore, the longitudinal Z-rings connect two canonical Z-rings.

260 And finally, the longitudinal septa could be formed with different spacing relative to the hyphal wall,

261 allowing asymmetric cleavage of spores. This strongly suggests that during normal Z-ring formation, a

262 second Z-ring is formed under different angles ranging from 45-90 degrees. Eventually, the longitudinal

263 septation also resulted in physical separation of spores along the horizontal axis, as seen by SEM and light

264 microscopy. This shows that these ectopic cell division events were completed via cytokinesis. We

265 previously showed that enhanced expression of SsgA, a cell division activator that assists in the localization

266 of SsgB, results in enhanced cell division and even the formation of ectopic spores in vegetative hyphae

267 (van Wezel et al., 2000). This underlines that SsgA and SsgB play a pivotal role in determining where septa

268 are positioned in the hyphae of streptomycetes.

Mutational and structural analysis, fluorescence imaging and 271 molecular dynamic simulation

270 of ScSsgB provided more insights into the structural basis for the observed longitudinal cell division. The

271 hydrogen bonds (E120-E120, E120-T119 and E120-G118, H121-Y35), salt bridges (E120-R55 and V115-R55)

272 and $\pi-\pi$ interaction (E120-Y35) stabilize and maintain the proper angle of the $\alpha 3$ helix. Substitutions in

273 E120 disrupts this critical interaction, and most likely results in major changes in the orientation of $\alpha 3$, by

274 up to 90 degrees. Mutations of the interacting partners all showed functional defects, as seen from the

275 blocked cell division (Y35H), septum rotation (V115G, G118A), DNA segregation (V115G, G118A) and

276 heterogeneity in spore sizes (V115G, G118A, H121I). Moreover, some longitudinal septa were seen in L96P 
mutants, which again can be explained by changes in the orientation of helix $\alpha 3$, as the mutation will

278 disrupt the interaction between the neighbouring $\alpha 2$ helix and $\beta 7$-strand. We therefore propose that

279 rotation of helix $\alpha 3$ is the driving factor for rotation of the Z-ring for up to 90 degrees along the long hyphal

280 axis, corresponding to the rotation of the septal plane seen in various mutants (Figure 7C). We propose

281 the following order of events leading to longitudinal cell division: (1) SsgB localizes to the septum sites and

282 recruits FtsZ, thereby assists in its polymerization and Z-ring formation; (2) in strains expressing specific

283 SsgB variants (particularly in G118, E120 and V96), an additional second Z-ring is formed perpendicular to

284 the canonical Z-rings, re-orienting the divisome to the central septum plane, parallel to the long axis of the

285 hyphae. This eventually results in diagonally or horizontally severed spores. SsgB G118V, E120G and E120A

286 had retained the ability to promote the assembly of FtsZ filaments in vitro. FRAP further confirmed the in

287 vivo studies by showing that the canonically localized foci and the centrally localized foci produced in SsgB

288 substitution mutants have the same dynamics, and that these are similar to those of wild-type SsgB.

So far, longitudinal fission had only been reported in studies by Bulgharesi and colleagues on the

291 hypermnestrae (Pende et al., 2018). Longitudinal cell division in these bacteria is host-polarized by their

292 nematode symbionts. The symbionts grow along the long axis and with increased cell width. The

293 machineries for growth and division are not reoriented; instead, they mesh 295 to a point where they

294 appear as "squeezed" E. coli cells (Pende et al., 2018). Our work shows that also in free-living bacteria, and

295 specifically in Streptomyces, cell division along the longitudinal axis is possible, caused by single aa

296 substitutions in the FtsZ-recruiting SsgB. It will be interesting to see if longitudinal division can also be

297 achieved in other bacteria, for example in bacteria where cell division is also positively controlled, such as

298 in Myxococcus xanthus. In Myxococcus, the ParA-like ATPase PomZ recruits FtsZ to midcell during

299 vegetative growth (Treuner-Lange et al., 2013). While this system is different from that controlled by SsgB, 
300 it will be worth investigating whether amino acid substitutions in PomZ may achieve similar changes in Z-

301 ring positioning.

302 In conclusion, our work shows that specific residues in SsgB, and especially residues G118 and

303 E120, play a key role in stabilizing the SsgB structure. Mutations in these residues result in major changes

304 in the control of Z-ring synthesis, resulting in additional septa that are formed diagonally or perpendicular

305 to the canonical septa, thereby severing spores in two halves. This underlines the crucial role of SsgB in

306 cell division control in streptomycetes.

\section{MATERIALS AND METHODS}

\section{Strains and culturing conditions}

310 All strains described in the paper are listed in Table S6. S. coelicolor M145 was obtained from the John

311 Innes centre strain collection. Its ssgB null mutant was published previously (Keijser et al., 2003).

312 Transformants harbouring SsgB-expression vectors based on the low-copy number shuttle vector pHJL401

313 (Larson and Hershberger, 1986) were grown on SFM agar plates containing $50 \mu \mathrm{g} / \mathrm{ml}$ apramycin (for the

$314 s s g B$ deletion) and $25 \mu \mathrm{g} / \mathrm{ml}$ thiostrepton (to maintain the plasmid) at $30^{\circ} \mathrm{C}$. For growth in liquid medium

315 the recombinants were grown in a 1:1 mix of TSBS and YEME at 300C. E. coli JM109 was used for

316 amplification of plasmids and E. coli Rosetta ${ }^{\text {TM }} 2$ (DE3) pLysS for overexpression and isolation of the His $_{6}$ -

317 tagged proteins.

\section{Random mutagenic PCR}

320 The SsgB promotor region and structural gene were amplified separately from the S. coelicolor

321 chromosome using oligonucleotide pairs pSsgB_fw + pSsgB_rv and SsgB_fw + SsgB_rv, respectively (Table

322 S7). An Ndel restriction site was introduced overlapping the translational start codon to enable ligation of

323 these fragments after mutagenic PCR. The $s s g B$ gene was cloned into a variant of pUC19 wherein the 
324 unique Ndel site had been removed, thereby creating pJPM1. Construct pJPM2 was based on the low-copy

325 number shuttle vector pHJL401 in which the original Ndel site had been removed, and contained the PCR-

326 amplified ssgB promotor fragment (cloned as EcoRI-HindIII fragment). Mutations in S. coelicolor ssgB were

327 introduced by random mutagenic PCR using pJPM1 as template, as described (Traag et al., 2007). The

328 mixture of mutagenized $s s g B$ genes produced by error-prone PCR was then ligated as Ndel-HindIII

329 fragments behind the natural ssgB promoter in pJPM2. The DNA was subsequently transformed into

330 protoplasts of the S. coelicolor ssgB null mutant, thereby generating a collection of Streptomyces colonies

331 each expressing a variant of SsgB from the natural ssgB promoter. Mutations were verified by DNA

332 sequencing.

\section{Scanner based imaging}

335 Plates were incubated at $30^{\circ} \mathrm{C}$ on a flat-bed CCD scanner and imaged every 30 min for 7 days. Automated 336 scanning was performed using Quickscan (www.burrotech.com) activated by the windows task scheduler.

337 Hereafter the image stack was analyzed for gray values using imageJ/FIJI. This was achieved by drawing an 338 equal sized circle in the middle of the grown colonies and measuring the grey level intensity of the stack 339 via the Measure stack plugin of ImageJ.

341 Automated spore measurements

342 For each image in the folder the scale is set to correspond to the microscopes settings, hereafter the image

343 size is increased to optimize for averaging of pixels values at later stages of the macro. After increasing the

344 image size by $5 \%$ in 20 consecutive operations the edge detection filter of imageJ is applied. Everything

345 above the default threshold is defined as a proper edge. The holes are filled and the spores and hyphae

346 that are detected in this manner are defined as "in focus". The original file is thresholded with default 
settings and combined with an AND operation with the sharp spores. Particles that fall within the range of spores are analyzed, with minimum size of $0.65 \mu \mathrm{m}^{2}$, and a roundness value between 0.75 and 1.

\section{Microscopy}

\section{Live/dead staining and DNA quantification}

352 For live/dead staining Streptomyces strains were grown on SFM agar plates, and after 7 days cover slips

353 were pressed onto the colony and mounted in PBS containing $10 \mu \mathrm{M}$ syto 9 and $10 \mu \mathrm{g} \cdot \mathrm{ml}^{-1}$ Propidium

354 iodide t. Fluorescence and light microscopy were performed as described previously (Willemse and van 355 Wezel, 2009).

For DNA quantification, Streptomyces colonies were grown against coverslips (at $45^{\circ}$ angle) on

357 SFM agar, and after 7 days taken out of the agar samples were fixed with 2\% paraformaldehyde for 5

358 minutes and washed with $70 \%$ ethanol. Subsequently spores were stained with $1 \mu \mathrm{M}$ Syto green

359 (ThermoFischer) and imaged with a Zeiss Axioplan 2, with 470/40 excitation and 525/50 emission. For

360 localization studies, cover slips were immediately imaged with either the same microscope was used for

361 imaging the SsgB-localization. For DNA quantification the total intensity of each separate spore in a spore

362 chain was measured, to circumvent staining variation the median value of each spore chain was set to 1

363 to normalize the data. To have enough data to normalize spore chains that were measured consisted of a

364 minimum of 10 spores. All images were background-corrected, setting the signal outside the hyphae to 0

365 to obtain a sufficiently dark background. Images were processed using Adobe Photoshop CS4 and FIJI.

367 Fluorescence recovery after photobleaching (FRAP)

368 FRAP was performed with a Zeiss Imager LSM 510, using $488 \mathrm{~nm}$ excitation and 505-550 $\mathrm{nm}$ detection as 369 described (Willemse et al., 2011). 
372 Cryo-scanning electron microscopy (cryo-SEM) was performed as described (Piette et al., 2005). For SEM

373 imaging of individual spores, impression prints of 7-day old confluent plates were obtained and fixed with

374 1.5\% glutaraldehyde in PBS. After 15 min fixation, samples were dehydrated using a graded series of

375 acetone $(70 \%, 80 \%, 90 \%, 96 \%, 100 \%, 15 \mathrm{~min}$ each) and subsequently critical point dried. Before

376 examination $10 \mathrm{~nm}$ Platinum/Palladium was sputter coated on the sample to prevent charging during

377 imaging. All images were obtained with a Jeol 7600 at $5 \mathrm{kV}$ and a working distance of $8 \mathrm{~mm}$.

378 For transmission electron microscopy (TEM), small cubes of colonies were fixed with $1.5 \%$

379 glutaraldehyde in PBS for 1 hour, postfixed with osmium tetroxide (1\%) for 1 hour, and dehydrated with a

380 graded ethanol series (70\%, 80\%, 90\%, 100\% 15 minutes each). Ultrathin sections of $70 \mathrm{~nm}$ were cut and

381 examined using a Jeol 1010. Purified FtsZ (15 $\mu \mathrm{M})$ was combined with an equimolar amount of wt SsgB,

382 or one of its mutants (SsgB E120G, SsgB E120A and SsgB $\Delta$ C) in a reaction buffer of 20 mM HEPES, pH 7.5,

$383150 \mathrm{mM} \mathrm{KCl}, 2.5 \mathrm{mM} \mathrm{MgCl}_{2}$. Polymerization was initiated by the addition of $2 \mathrm{mM}$ GTP to the assembly

384 reaction. $50 \mu$ reaction mixture was incubated for 2 minutes at $37^{\circ} \mathrm{C} .15 \mu \mathrm{l}$ aliquot was placed on a carbon

385 coated copper grid and negatively stained with $2 \%$ uranyl acetate for 10 minutes, then washed and dried.

386 Images were collected using transmission electron microscope (Jeol 1010) operated at $70 \mathrm{kV}$ with 670

$387 \mathrm{pA} \cdot \mathrm{cm}^{-2}$ density and recorded on a camera.

389 Co-pelleting assay

390 For pelleting experiments, purified FtsZ, with or without equimolar ratios of wt SsgB, or its mutants (SsgB

391 E120G, SsgB E120A and SsgB $\Delta$ C) were mixed at a final concentration of $15 \mu \mathrm{M}$. The samples were pre-spun

392 at 45 k r.p.m in a Beckman 40.1 rotor for 30 minutes. Then supernatants were transferred to new tubes

393 and $2 \mathrm{mM} \mathrm{MgCl}_{2}$ and GTP were added. After $10 \mathrm{~min}$ of incubation at $30^{\circ} \mathrm{C}$, the tubes were centrifuged at

$39445 \mathrm{k} \mathrm{r.p.m}$ at $20^{\circ} \mathrm{C}$ for 30 minutes. the supernatants were removed for analysis and pellets were washed 
with the same buffer aside from the proteins. Pellets were dissolved in SDS gel loading buffer. All the samples were analyzed on a $4 \%-16 \%$ Pre-cast SDS-PAGE gel (BIO-RAD).

\section{Protein expression and purification}

399 For expression of $\mathrm{His}_{6}$-tagged fusions of SsgB and FtsZ in E. coli, the entire coding region of $s s g B$ or its 400 mutants were cloned as Ndel-HindIII fragments into pET28a (Novagen). Sequences of S. coelicolor ftsZ 401 were codon optimized prior to cloning into pET28a. SsgB was overexpressed in E. coli Rosetta ${ }^{\text {TM }} 2$ (DE3) 402 pLys strain as a 157 aa-long fusion protein containing the 137 aa native polypeptide and an $\mathrm{N}$-terminal 403 tag, GSSHHHHHHSSG. SsgB point mutants E120G and E120A, or its deletion mutant ( $\Delta$ SsgB, 1-114 aa; 404 lacking the C-terminal 11 aa), were expressed in the same way. FtsZ was produced as C-terminal His-tag 405 fusion. His 6 -tagged FtsZ proteins were purified using routine methods as described (Mahr et al., 2000).

\section{Oligomerization studies of SsgB}

408 The polymerization state of SsgB was analyzed by size-exclusion chromatography on an ÄKTA-pure system 409 using column Superdex ${ }^{\mathrm{TM}} 75$ Increase 10/300 GL and Superdex ${ }^{\mathrm{TM}} 75$ Increase 5/150 GL at room 410 temperature, respectively. The elution solvent was $20 \mathrm{mM} \mathrm{HEPES}, 150 \mathrm{mM} \mathrm{KCl}, 2.5 \mathrm{mM} \mathrm{MgCl}$, $5 \%$ Glycerol,

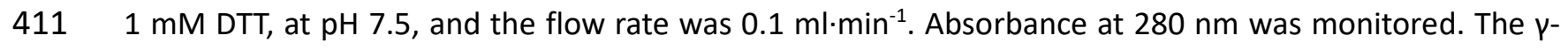
412 globulin, conalbumin, ovalbumin, myoglobin, and vitamin B12 were used as molecular weight standards.

\section{Crystallization and structure determination}

415 SsgB trimer crystals were grown at $18^{\circ} \mathrm{C}$ by using the sitting-drop vapor-diffusion method. In a typical 416 experiment, $1 \mu \mathrm{l}$ of the protein stock $\left(6.2 \mathrm{mg} \cdot \mathrm{ml}^{-1}\right.$ protein) was mixed with $1 \mu \mathrm{l}$ of a reservoir buffer 417 consisting of $0.2 \mathrm{M}$ Sodium chloride, $0.1 \mathrm{M}$ Sodium/potassium phosphate (pH 6.2), 50\% PEG200. Crystals 418 were moved to the same condition supplemented with $20 \%$ glycerol and flash-frozen in liquid nitrogen. 
Data were collected at beam line ID30B (McCarthy et al., 2018) at ESRF (Grenoble, France, 2017). Images

420 were collected with a $0.15^{\circ}$ oscillation angle and an exposure time of $0.037 \mathrm{~s}$ per frame at $100 \mathrm{~K}$. Crystals

421 forms diffracted to $2.1 \AA \AA$ for SsgB trimer. The data was processed with XDS (Kabsch, 2010) and scaled using

422 AIMLESS (Evans and Murshudov, 2013) from CCP4 package(Bailey, 1994). Phases of SsgB trimer were

423 calculated by molecular replacement with SsgB ${ }^{\text {Tfus }}$ PDB entry $1 \mathrm{C} 3 \mathrm{M}(\mathrm{Xu}$ et al., 2009) as a model using

424 MOLREP (Vagin and Teplyakov, 1997). The structures were finalized by manual building in COOT (Emsley

425 et al., 2010) and refined with REFMAC (Murshudov et al., 1997). Residues were in the most favored regions

426 of the Ramachandran plot (Ramachandran et al., 1963) as determined by PROCHECK (Laskowski et al.,

427 1993). Crystallographic data are summarized in Table 2. SsgB trimer structure was deposited in the PDB

428 with code 6SLD, respectively.

\section{Molecular simulation}

431 All atom molecular dynamics (AAMD) simulation of SsgB wt and SsgB E120G were prepared and run using

432 Gromacs 2016 package (Hess et al., 2008). The simulated protein, wide type or mutant type, was centered

433 in a cubic box surrounded by water molecules and counter ions $\mathrm{Na}^{+}$were added to keep the total charge

434 of the simulation box to be zero. The bonded and non-bonded parameters were obtained from

435 AMBER99SB force filed (Hornak et al., 2006). The SPCE (Berendsen et al., 1987) water model was used.

436 The Particle-mesh Ewald method was used to treat the long-range electrostatic interactions (Darden et al.,

437 1993). A cutoff of $12 \AA$ was used for non-bonded interactions. Temperature was maintained at $300 \mathrm{~K}$ using

438 v-rescale thermostat (Bussi et al., 2009); Pressure was maintained at 1 bar Parrinello-Rahman barostat

439 (Parrinello and Rahman, 1981; Rahman and Stilling.Fh, 1971). The simulation box was cubic of length

440 around $9.2 \mathrm{~nm}$, with periodic boundary conditions applied to all dimensions. All simulated systems were

441 stabilized though energy minimization, short NVT (10 ps) and short NPT (5 ns) MD simulations to relax to 
favorable conformations. NPT simulations at $300 \mathrm{~K}$ and 1.0 bar were performed upon the stabilized

443 structures and 50 ns trajectories were collected for our analysis.

\section{ACKNOWLEDGEMENTS}

446 We gratefully thank to the European Synchrotron Radiation Facility, Grenoble, France. This work was supported by a grant from the Chinese Scholarship Council (CSC) to X.X.

450 The authors declare no conflict of interests.

\section{REFERENCES}

Adams, D.W., and Errington, J. (2009) Bacterial cell division: assembly, maintenance and disassembly of the Z ring. Nat Rev Microbiol 7: 642-653.

Bailey, S. (1994) The Ccp4 Suite - Programs for Protein Crystallography. Acta Crystallogr D 50: 760-763.

Barka, E.A., Vatsa, P., Sanchez, L., Gavaut-Vaillant, N., Jacquard, C., Meier-Kolthoff, J., Klenk, H.P., Clément, C., Oudouch, Y., and van Wezel, G.P. (2016) Taxonomy, physiology, and natural products of the Actinobacteria. Microbiol Mol Biol Rev 80: 1-43.

Berendsen, H.J.C., Grigera, J.R., and Straatsma, T.P. (1987) The Missing Term in Effective Pair Potentials. J Phys Chem 91: 6269-6271.

Bussi, G., Zykova-Timan, T., and Parrinello, M. (2009) Isothermal-isobaric molecular dynamics using stochastic velocity rescaling. J Chem Phys 130: 074101.

Celler, K., Koning, R.I., Willemse, J., Koster, A.J., and van Wezel, G.P. (2016) Cross-membranes orchestrate compartmentalization and morphogenesis in Streptomyces. Nat Commun 7: 11836.

Claessen, D., Rozen, D.E., Kuipers, O.P., Sogaard-Andersen, L., and van Wezel, G.P. (2014) Bacterial solutions to multicellularity: a tale of biofilms, filaments and fruiting bodies. Nat Rev Microbiol 12: 115-124.

Darden, T., York, D., and Pedersen, L. (1993) Particle Mesh Ewald - an N.Log(N) Method for Ewald Sums in Large Systems. J Chem Phys 98: 10089-10092.

Emsley, P., Lohkamp, B., Scott, W.G., and Cowtan, K. (2010) Features and development of Coot. Acta Crystallogr D 66: 486-501.

Evans, P.R., and Murshudov, G.N. (2013) How good are my data and what is the resolution? Acta Crystallogr D 69: 1204-1214. 
Flärdh, K., and Buttner, M.J. (2009) Streptomyces morphogenetics: dissecting differentiation in a filamentous bacterium. Nat Rev Microbiol 7: 36-49.

Girard, G., Traag, B.A., Sangal, V., Mascini, N., Hoskisson, P.A., Goodfellow, M., and van Wezel, G.P. (2013) A novel taxonomic marker that discriminates between morphologically complex actinomycetes. Open Biol 3: 130073.

Hale, C.A., and deBoer, P.A.J. (1997) Direct binding of FtsZ to ZipA, an essential component of the septal ring structure that mediates cell division in E-coli. Cell 88: 175-185.

Hess, B., Kutzner, C., van der Spoel, D., and Lindahl, E. (2008) GROMACS 4: Algorithms for highly efficient, load-balanced, and scalable molecular simulation. J Chem Theory Comput 4: 435-447.

Hopwood, D.A. (2007) Streptomyces in nature and medicine: the antibiotic makers. New York: Oxford University Press.

Hornak, V., Abel, R., Okur, A., Strockbine, B., Roitberg, A., and Simmerling, C. (2006) Comparison of multiple amber force fields and development of improved protein backbone parameters. Proteins 65: 712-725.

Jakimowicz, D., and van Wezel, G.P. (2012) Cell division and DNA segregation in Streptomyces: how to build a septum in the middle of nowhere? Mol Microbiol 85: 393-404.

Kabsch, W. (2010) Xds. Acta Crystallogr D 66: 125-132.

Keijser, B.J., Noens, E.E., Kraal, B., Koerten, H.K., and van Wezel, G.P. (2003) The Streptomyces coelicolor ssgB gene is required for early stages of sporulation. FEMS Microbiol Lett 225: 59-67.

Kelemen, G.H., Brian, P., Flärdh, K., Chamberlin, L., Chater, K.F., and Buttner, M.J. (1998) Developmental regulation of transcription of whiE, a locus specifying the polyketide spore pigment in Streptomyces coelicolor A3 (2). J Bacteriol 180: 2515-2521.

Larson, J.L., and Hershberger, C.L. (1986) The minimal replicon of a streptomycete plasmid produces an ultrahigh level of plasmid DNA. Plasmid 15: 199-209.

Laskowski, R.A., Macarthur, M.W., Moss, D.S., and Thornton, J.M. (1993) Procheck - a Program to Check the Stereochemical Quality of Protein Structures. J Appl Crystallogr 26: 283-291.

Li, Y., Hsin, J., Zhao, L.Y., Cheng, Y.W., Shang, W.N., Huang, K.C., Wang, H.W., and Ye, S. (2013) FtsZ Protofilaments Use a Hinge-Opening Mechanism for Constrictive Force Generation. Science 341: 392-395.

Lluch-Senar, M., Querol, E., and Pinol, J. (2010) Cell division in a minimal bacterium in the absence of ftsZ. Mol Microbiol 78: 278-289.

Lutkenhaus, J. (2007) Assembly dynamics of the bacterial MinCDE system and spatial regulation of the $\mathrm{Z}$ ring. Annu Rev Biochem 76: 539-562.

Mahr, K., van Wezel, G.P., Svensson, C., Krengel, U., Bibb, M.J., and Titgemeyer, F. (2000) Glucose kinase of Streptomyces coelicolor A3(2): large-scale purification and biochemical analysis. Antonie Van Leeuwenhoek 78: 253-261.

Margolin, W. (2005) FtsZ and the division of prokaryotic cells and organelles. Nat Rev Mol Cell Biol 6: 862-871.

McCarthy, A.A., Barrett, R., Beteva, A., Caserotto, H., Dobias, F., Felisaz, F., Giraud, T., Guijarro, M., Janocha, R., Khadrouche, A., Lentini, M., Leonard, G.A., Marrero, M.L., MalbetMonaco, S., McSweeney, S., Nurizzo, D., Papp, G., Rossi, C., Sinoir, J., Sorez, C., Surr, J., Svensson, O., Zander, U., Cipriani, F., Theveneau, P., and Mueller-Dieckmann, C. (2018) 
ID30B-a versatile beamline for macromolecular crystallography experiments at the ESRF. J Synchrotron Radiat 25: 1249-1260.

McCormick, J.R., Su, E.P., Driks, A., and Losick, R. (1994) Growth and viability of Streptomyces coelicolor mutant for the cell division gene ftsZ. Mol Microbiol 14: 243-254.

McCormick, J.R. (2009) Cell division is dispensable but not irrelevant in Streptomyces. Curr Opin Biotechnol 12: 689-698.

Murshudov, G.N., Vagin, A.A., and Dodson, E.J. (1997) Refinement of macromolecular structures by the maximum-likelihood method. Acta Crystallogr D 53: 240-255.

Noens, E.E., Mersinias, V., Traag, B.A., Smith, C.P., Koerten, H.K., and van Wezel, G.P. (2005) SsgAlike proteins determine the fate of peptidoglycan during sporulation of Streptomyces coelicolor. Mol Microbiol 58: 929-944.

Parrinello, M., and Rahman, A. (1981) Polymorphic Transitions in Single-Crystals - a New Molecular-Dynamics Method. J Appl Phys 52: 7182-7190.

Pende, N., Wang, J., Weber, P.M., Verheul, J., Kuru, E., Rittmann, S.K.R., Leisch, N., VanNieuwenhze, M.S., Brun, Y.V., den Blaauwen, T., and Bulgheresi, S. (2018) HostPolarized Cell Growth in Animal Symbionts. Curr Biol 28: 1039-1051 e1035.

Pichoff, S., and Lutkenhaus, J. (2002) Unique and overlapping roles for ZipA and FtsA in septal ring assembly in Escherichia coli. EMBO J 21: 685-693.

Pichoff, S., and Lutkenhaus, J. (2005) Tethering the $Z$ ring to the membrane through a conserved membrane targeting sequence in FtsA. Mol Microbiol 55: 1722-1734.

Piette, A., Derouaux, A., Gerkens, P., Noens, E.E., Mazzucchelli, G., Vion, S., Koerten, H.K., Titgemeyer, F., De Pauw, E., Leprince, P., van Wezel, G.P., Galleni, M., and Rigali, S. (2005) From dormant to germinating spores of Streptomyces coelicolor $A 3(2)$ : new perspectives from the crp null mutant. J Proteome Res 4: 1699-1708.

Rahman, A., and Stilling.Fh (1971) Molecular Dynamics Study of Liquid Water. J Chem Phys 55: 3336-+.

Ramachandran, G.N., Ramakrishnan, C., and Sasisekharan, V. (1963) Stereochemistry of Polypeptide Chain Configurations. J Mol Biol 7: 95-\&.

Shapiro, L., McAdams, H.H., and Losick, R. (2009) Why and How Bacteria Localize Proteins. Science 326: 1225-1228.

Szwedziak, P., Wang, Q., Bharat, T.A., Tsim, M., and Lowe, J. (2014) Architecture of the ring formed by the tubulin homologue FtsZ in bacterial cell division. Elife 3: e04601.

Traag, B.A., Seghezzi, N., Vijgenboom, E., and van Wezel, G.P. (2007) Characterization of the sporulation control protein SsgA by use of an efficient method to create and screen random mutant libraries in streptomycetes. Appl Environ Microbiol 73: 2085-2092.

Traag, B.A., and van Wezel, G.P. (2008) The SsgA-like proteins in actinomycetes: small proteins up to a big task. Antonie Van Leeuwenhoek 94: 85-97.

Treuner-Lange, A., Aguiluz, K., van der Does, C., Gomez-Santos, N., Harms, A., Schumacher, D., Lenz, P., Hoppert, M., Kahnt, J., Munoz-Dorado, J., and Sogaard-Andersen, L. (2013) PomZ, a ParA-like protein, regulates Z-ring formation and cell division in Myxococcus xanthus. Mol Microbiol 87: 235-253.

Vagin, A., and Teplyakov, A. (1997) MOLREP: an automated program for molecular replacement. J Appl Crystallogr 30: 1022-1025. 
van der Heul, H.U., Bilyk, B.L., McDowall, K.J., Seipke, R.F., and van Wezel, G.P. (2018) Regulation of antibiotic production in Actinobacteria: new perspectives from the post-genomic era. Nat Prod Rep 35: 575-604.

van Dissel, D., Claessen, D., and Van Wezel, G.P. (2014) Morphogenesis of Streptomyces in submerged cultures. Adv Appl Microbiol 89: 1-45.

van Wezel, G.P., van der Meulen, J., Kawamoto, S., Luiten, R.G., Koerten, H.K., and Kraal, B. (2000) $s s g A$ is essential for sporulation of Streptomyces coelicolor A3(2) and affects hyphal development by stimulating septum formation. J Bacteriol 182: 5653-5662.

Vijgenboom, E., Woudt, L.P., Heinstra, P.W., Rietveld, K., van Haarlem, J., van Wezel, G.P., Shochat, S., and Bosch, L. (1994) Three tuf-like genes in the kirromycin producer Streptomyces ramocissimus. Microbiology 140 ( Pt 4): 983-998.

Wildermuth, H., and Hopwood, D. (1970) Septation during sporulation in Streptomyces coelicolor. J Gen Microbiol 60: 51-59.

Willemse, J., and van Wezel, G.P. (2009) Imaging of Streptomyces coelicolor A3(2) with Reduced Autofluorescence Reveals a Novel Stage of FtsZ Localization. PLoS ONE 4: e4242.

Willemse, J., Borst, J.W., de Waal, E., Bisseling, T., and van Wezel, G.P. (2011) Positive control of cell division: FtsZ is recruited by SsgB during sporulation of Streptomyces. Genes Dev 25: 89-99.

Xu, Q., Traag, B.A., Willemse, J., McMullan, D., Miller, M.D., Elsliger, M.A., Abdubek, P., Astakhova, T., Axelrod, H.L., Bakolitsa, C., Carlton, D., Chen, C., Chiu, H.J., Chruszcz, M., Clayton, T., Das, D., Deller, M.C., Duan, L., Ellrott, K., Ernst, D., Farr, C.L., Feuerhelm, J., Grant, J.C., Grzechnik, A., Grzechnik, S.K., Han, G.W., Jaroszewski, L., Jin, K.K., Klock, H.E., Knuth, M.W., Kozbial, P., Krishna, S.S., Kumar, A., Marciano, D., Minor, W., Mommaas, A.M., Morse, A.T., Nigoghossian, E., Nopakun, A., Okach, L., Oommachen, S., Paulsen, J., Puckett, C., Reyes, R., Rife, C.L., Sefcovic, N., Tien, H.J., Trame, C.B., van den Bedem, H., Wang, S., Weekes, D., Hodgson, K.O., Wooley, J., Deacon, A.M., Godzik, A., Lesley, S.A., Wilson, I.A., and van Wezel, G.P. (2009) Structural and functional characterizations of SsgB, a conserved activator of developmental cell division in morphologically complex actinomycetes. J Biol Chem 284: 25268-25279.

Yagüe, P., Willemse, J., Koning, R.I., Rioseras, B., Lopez-Garcia, M.T., Gonzalez-Quinonez, N., Lopez-Iglesias, C., Shliaha, P.V., Rogowska-Wrzesinska, A., Koster, A.J., Jensen, O.N., van Wezel, G.P., and Manteca, A. (2016) Subcompartmentalization by cross-membranes during early growth of Streptomyces hyphae. Nat Commun 7: 12467.

Zhang, L., Willemse, J., Claessen, D., and van Wezel, G.P. (2016) SepG coordinates sporulationspecific cell division and nucleoid organization in Streptomyces coelicolor. Open Biol 6: 150164. 
601 Table 1. Data collection, model refinement, and final structure statistics.

602

\begin{tabular}{|c|c|}
\hline & SsgB trimer \\
\hline \multicolumn{2}{|l|}{ Data collection } \\
\hline PDB entry & 6SLC \\
\hline X-ray source & ESRF ID30B \\
\hline Space group & 14 \\
\hline Unit cell (Å) & $a=b=155.22$ \\
\hline$a, b, c$ & $c=53.85$ \\
\hline$\alpha / \beta / \gamma$ & $90.0 / 90.0 / 90.0$ \\
\hline Wavelength $(\AA ̊)$ & 0.9750 \\
\hline \multirow[t]{2}{*}{ Resolution $(\AA ̊)$} & 109.93-2.27 \\
\hline & $(2.31-2.27)$ \\
\hline No. unique reflections & 33201 \\
\hline Multiplicity & $4.0(4.1)$ \\
\hline Completeness (\%) & $95.5(92.1)$ \\
\hline Mean $\left(<1>\mid<\sigma_{1}>\right)$ & $9.4(1.1)$ \\
\hline$R_{\text {merge }}(\%)^{a}$ & 6.5 (100.9) \\
\hline Wilson B-factor $\left(\AA^{2}\right)$ & 47.9 \\
\hline \multicolumn{2}{|l|}{ Refinement } \\
\hline R factor (all reflections) (\%) & 21.0 \\
\hline Free R factor $(\%)^{b}$ & 25.6 \\
\hline Number of atoms & 3138 \\
\hline Number of water molecules & 136 \\
\hline Number of other molecules & $1 \mathrm{PO}_{4}{ }^{3-} ; 6 \mathrm{PEG} ; 14 \mathrm{GOL}$ \\
\hline RMSD bond lengths $(\AA ̊)$ & 0.007 \\
\hline RMSD bond angles $\left({ }^{\circ}\right)$ & 1.413 \\
\hline \multicolumn{2}{|l|}{ Ramachandran Plot } \\
\hline Favored regions (\%) & 355 (95.95) \\
\hline Allowed regions (\%) & $15(4.05)$ \\
\hline Outliers (\%) & $0(0)$ \\
\hline
\end{tabular}

Values in parenthesis are for the highest resolution shell.

$604{ }^{a} R_{\text {merge }}=\left[\sum_{h k l} \sum_{i}\left|l_{i}(h k l)-<l(h k l)>\right|\right] /\left[\sum_{h k l} \sum_{i} l_{i}(h k l)\right]$, where $l_{i}(h k l)$ is the $i^{\text {th }}$ measurement of 605 reflection, $h k l$ and $\langle l(h k l)>$ is the weighted mean of all measurements of $h k l$.

606 b In the free $\mathrm{R}$ factor calculations, 5\% of the reflections were used. 
610 Figure 1. Transmission electron micrographs of sporogenic hyphae from the WT strain and from

611 transformants expressing SsgB substitution mutants. (A) SsgB substitution mutants resulted in pleiotropic

612 sporulation defects. T66A showed normal sporulation; D70G, S76A, E92G, S106A resulted in thinner cell

613 walls; D56G, L88R, L96R and L96P affected DNA condensation and/or segregation; L96P, V115G, G118V,

614 E120G gave rise to the formation of additional diagonal or longitudinal septa. V15A, S16P, E18G, D30Y,

615 T31A, T31M, H38R, V49G, W51R, L62P, H63L, V83A, E94G, E105G, Q128R, S131A all showed highly variable

616 spore sizes (see also Table S2). Bars: $500 \mathrm{~nm}$ for all TEM micrographs. (B) TEM images of spore chains from

617 six additional transformants expressing substitution mutants of SsgB (E120F, H, I, K, L or N) that also gave 618 rise to longitudinal division (for SsgB E120G see Fig. 1A).

620 Figure 2. Impression prints of spores from cells expressing wild-type SsgB or SsgB E120 mutants. (A) SEM

621 imaging. The fixation procedure led to occasional artefacts in the form of mild damage to spores (panels

622 a, c) and sometimes to collapse of spores (panel d). However, the collapsed spores can clearly be

623 discriminated from those that had been divided along the horizontal axis (panel e, f). (B) Longitudinal cell

624 division (pointed by arrowhead) revealed by fluorescent microscope for different E120 mutants. Left,

625 bright-field images; middle, Syto9/PI stained images; right, overlays of the two images. All the spores were

626 obtained after 7 days of growth from wild-type cells or from transformants of its $\operatorname{ssg} B$ null mutant

627 expressing SsgB E120 mutants. Bars, $1 \mu \mathrm{m}$.

629 Figure 3. Intensity plots of SsgB foci on the septa. (A) The wild-type SsgB localization during early cell

630 division shows two foci on either side of the hyphal wall; (B) Mutants expressing SsgB(G118V) or

631 SsgB(E120G) showed aberrant localization, whereby SsgB was located all over the hyphal wall; (C)

632 Occasional longitudinal septation was seen, whereby eGFP fusions of SsgB mutant proteins localized 
parallel to the hyphal wall and in the middle of the hyphae. The red box indicates 630 the width of the box

634 that was used to produce the profiles.

Figure 4. Co-localization and interactions between SsgB substitution mutants and FtsZ. (A) FtsZ

637 sedimentation assay with different SsgB variants, in the presence of either GTP or GDP. Note that GTP is

638 required for sedimentation. Initial samples (I) were used to assess the total protein content in each

639 reaction. Soluble $(S)$ and pelleted $(P)$ fractions were separated by centrifugation at $45,000 \mathrm{rpm}$. (B) TEM

640 images of FtsZ filament structures formed in the presence of GTP and different SsgB variants: (a) FtsZ alone;

641 (b, c) FtsZ with wt SsgB; (d) FtsZ with SsgBDC; (e) FtsZ with SsgB E120G; (f) FtsZ with SsgB E120A.

Figure 5. Crystal structure of the ScSsgB trimer. (A) Ribbon diagrams showing the monomer structure of

644 SsgB from S. coelicolor. (B) The overall structure of ScSsgB reveals a trimer. Structure statistics are listed in

645 Table 2. The interface between adjacent monomers is formed by two antiparallel $\beta$-sheets. (C) The 646 monomer structure of SsgB from T. fusca (PDB code 3CM1). (D) The interface between adjacent monomers

647 of TfSsgB is formed by $\alpha$-helices. (E) Overlap of ScSsgB (blue) and TfSsgB (orange) subunits. Left, side view 648 of the electrostatic surface alignment of ScSsgB and TfSsgB structure. Right, the same electrostatic figure 649 but rotated by $180^{\circ}$.

651 Figure 6. Key mutations and their interactions in SsgB trimer structure. (A) Left, mutant residues L96,

$652 \underline{\mathrm{G} 118}, \underline{\mathrm{E} 120}$ (marine) which showed tilted division are highlighted; Mutation of residues $\underline{\mathrm{Y} 35}, \mathrm{~V} 37, \underline{\mathrm{L} 57} \underline{\underline{\mathrm{L} 97}}$

653 (deep salmon) resulted in a sporulation block; mutation of residues D70, S76, R122 (light orange) led to 654 thinner cell walls; variants D56, L96 and L88 (violet purple) had damaged DNA. Middle, Right, top view of 655 all the functional mutants mapped on the surface structures. Conserved residues are underlined. (B) Key 656 mutations (V115, G118 and E120) are clustered on the lid of the $\beta$-barrel, consisting of $\alpha 1, \alpha 2-\alpha 3$ and $\beta 1$ - 
$657 \quad \beta 2$ loops. (C) Stereo view of E120 in the monomer structure and its interactions with the surrounding 658 residues.

659

660 Figure 7. Molecular simulation of SsgB wt and E120G mutant. (A) MD result of SsgB wt structure. The $\alpha 3$

661 helix stays at the same orientation and the distance between R55 and E120 keeps at $2.8 \AA$ and $3.0 \AA$, before

662 (green) and after MD (grey). (B) The arrows indicate the changed angle of SsgB E120G mutant (cyan)

663 compared to the wild type SsgB (grey) after MD. (C) Model for how E120G mutant enhances longitudinal

664 cell division.

665

666 


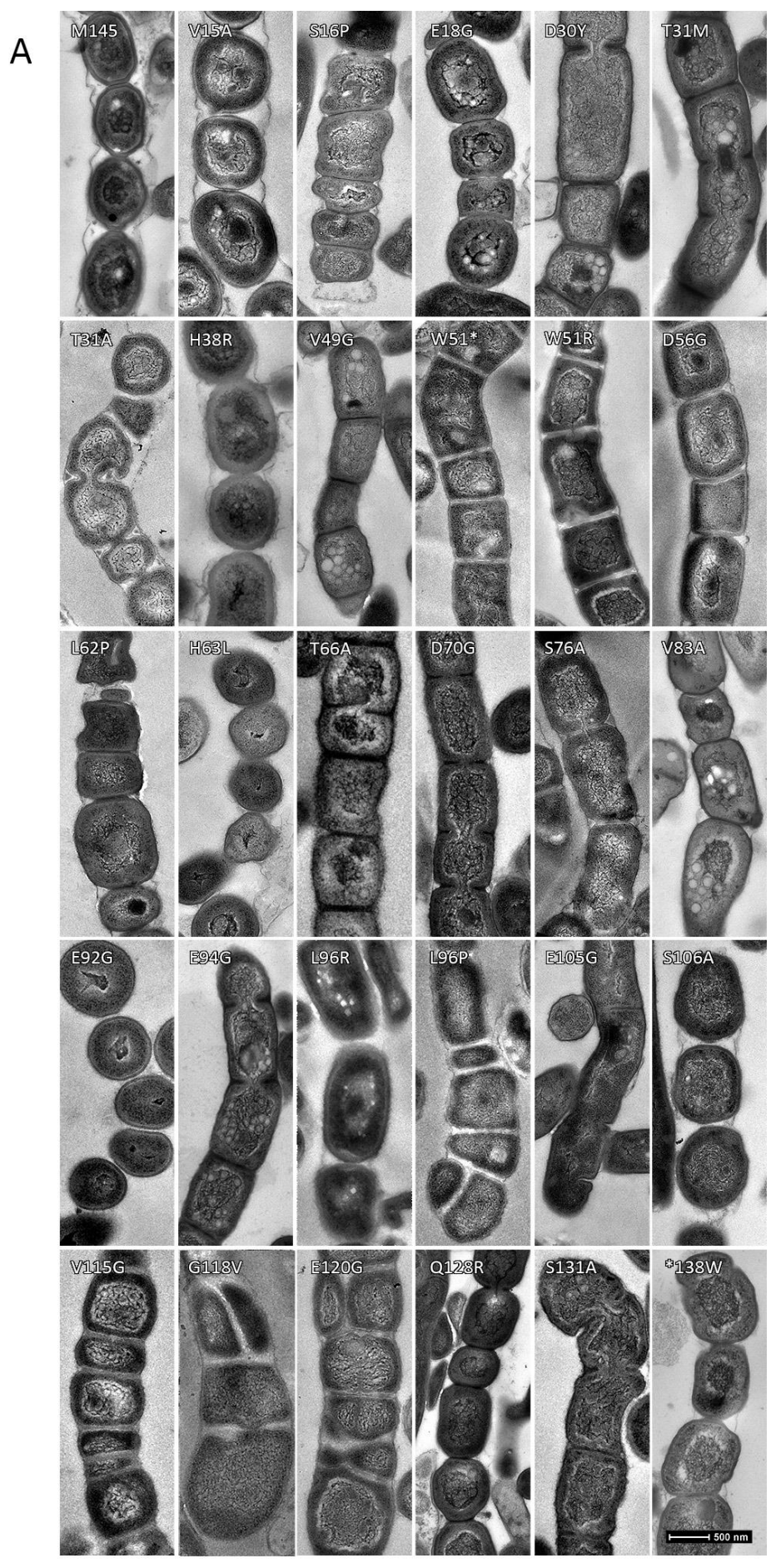

B

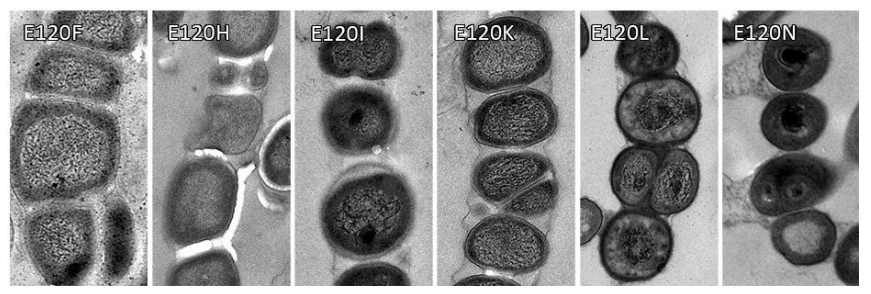

Figure 1 

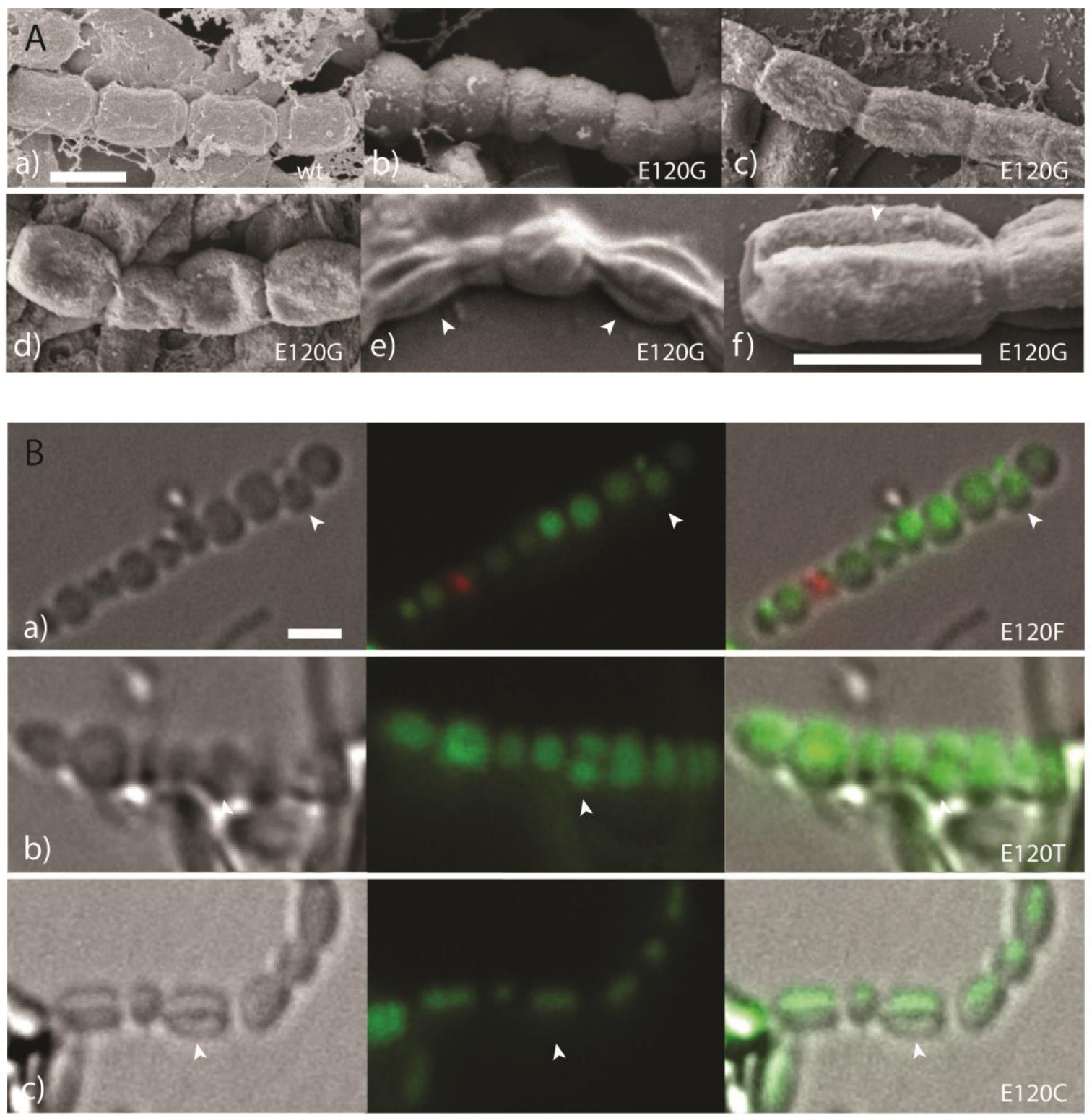

Figure 2 


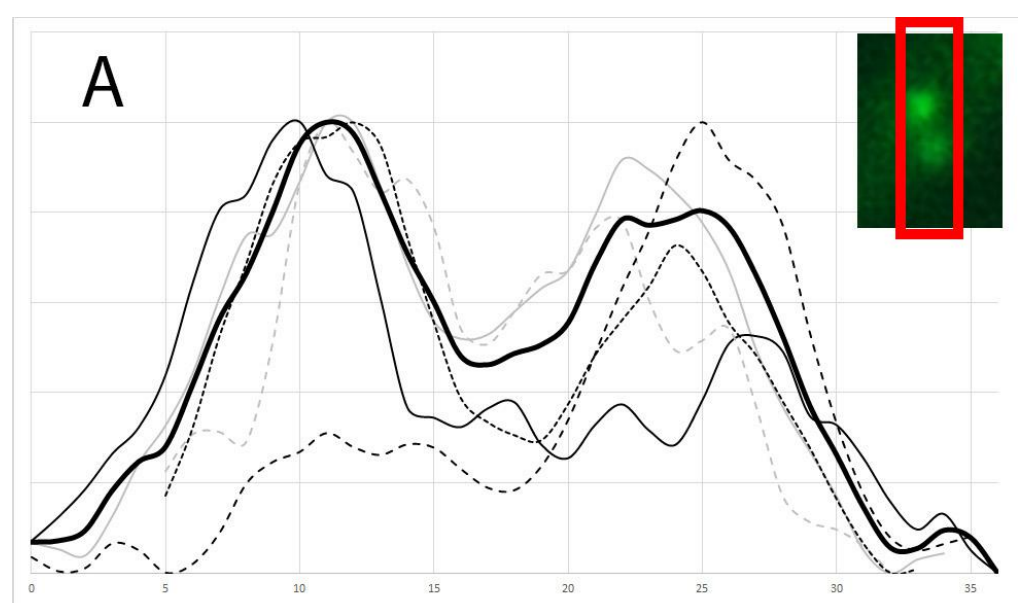

-Y1 —Y2 -.Y3 --.Y4 ---YY5 -Average

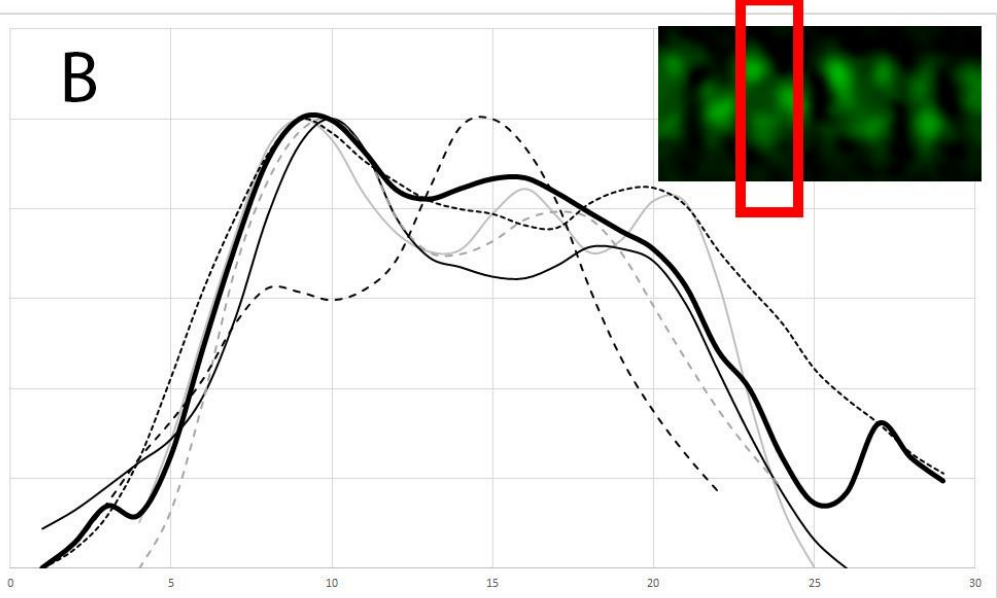

-Y1 -Y2 ---Y3 ---Y4 ---.Y5 —Average

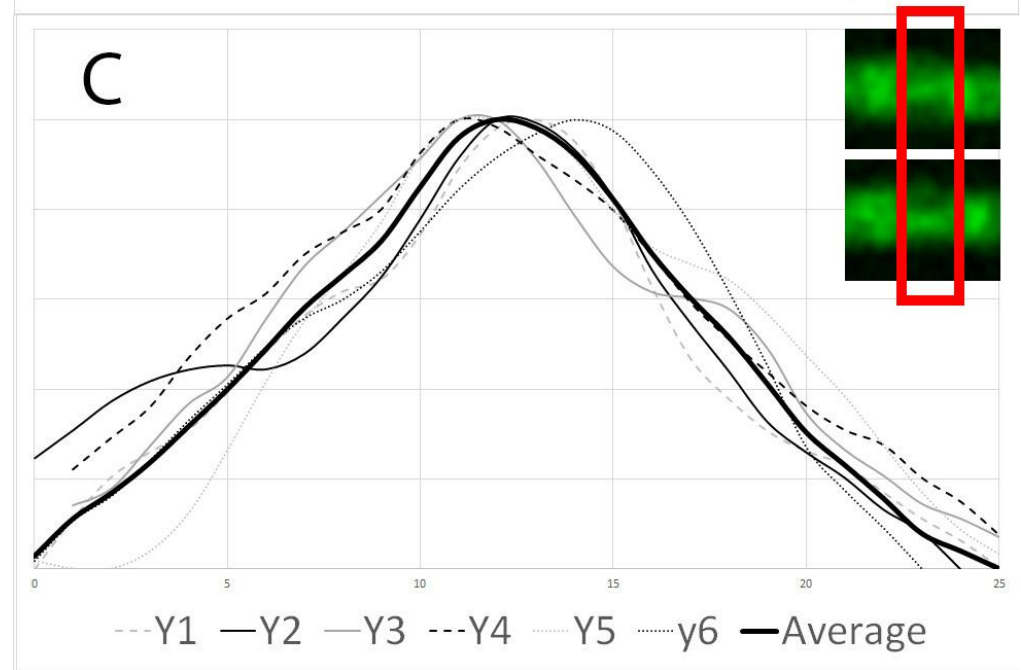

Figure 3 


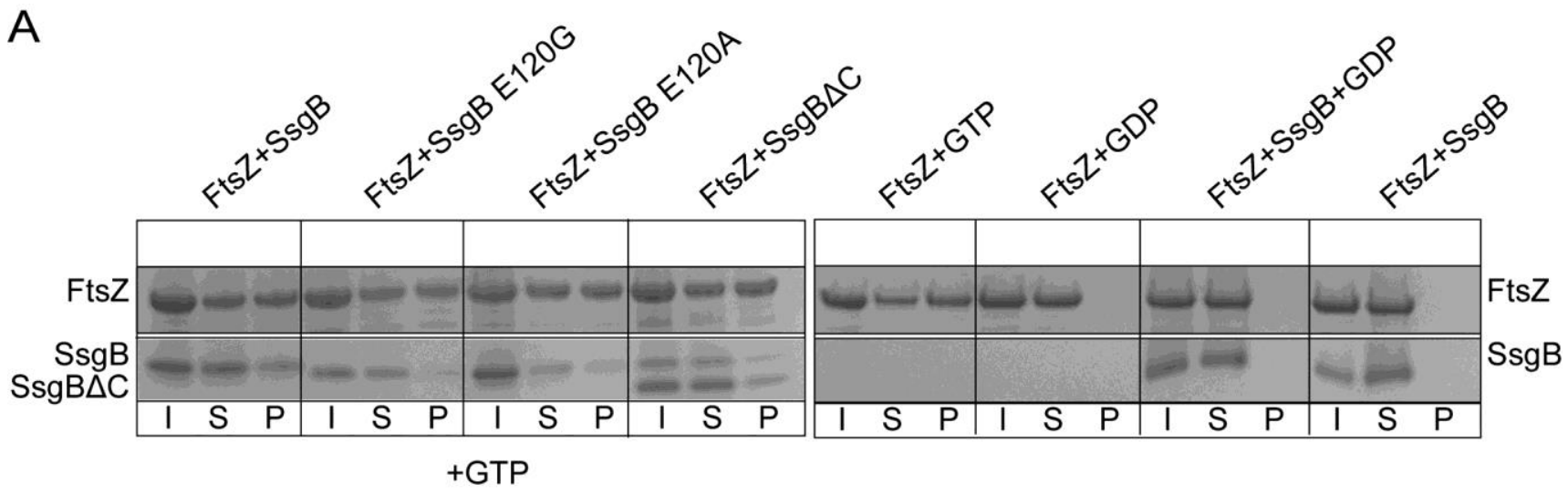

B
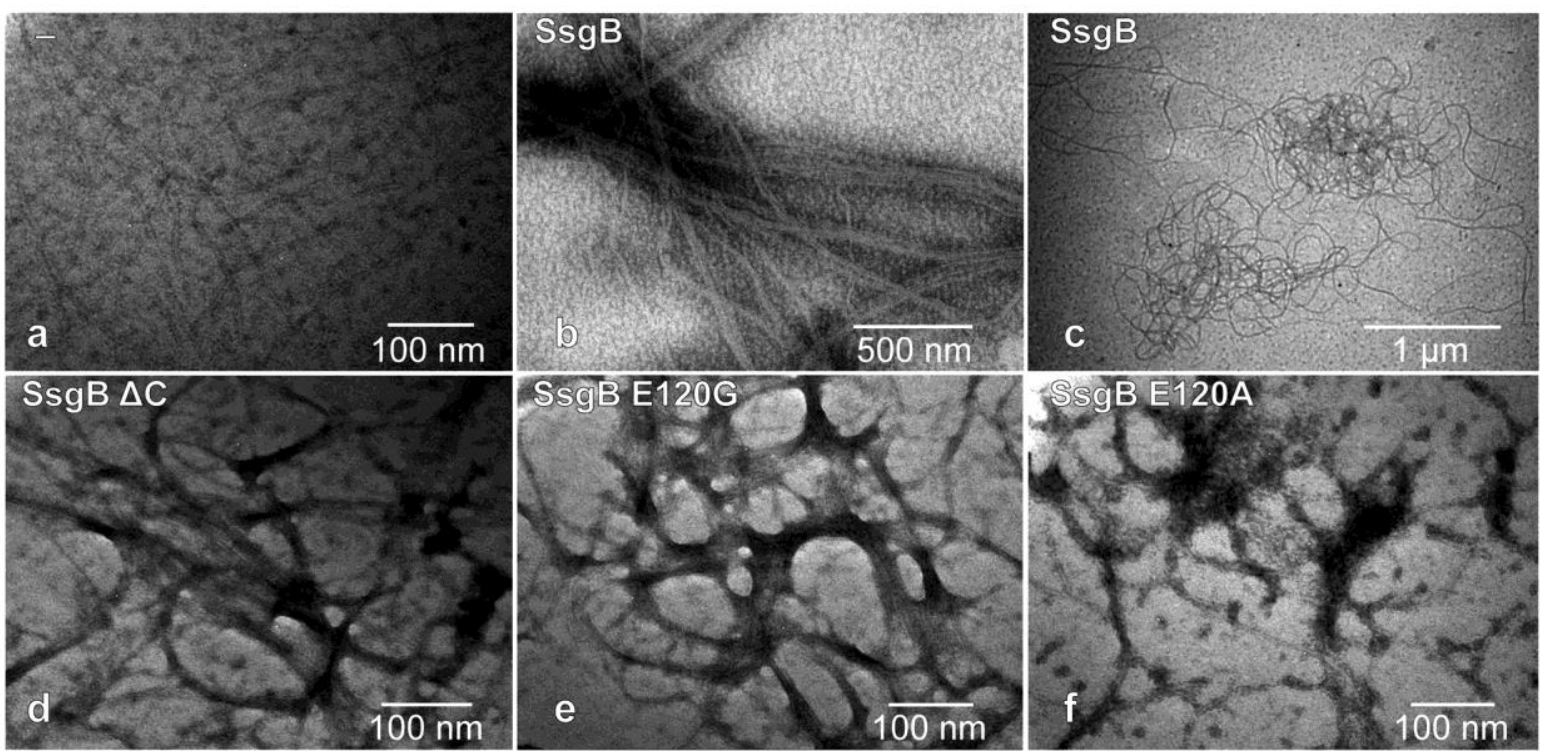

Figure 4 
A

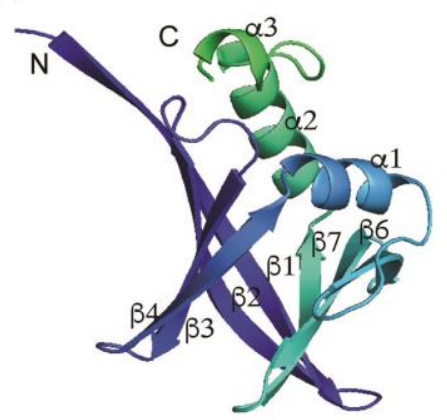

C

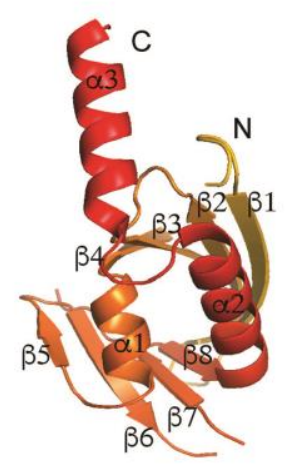

E

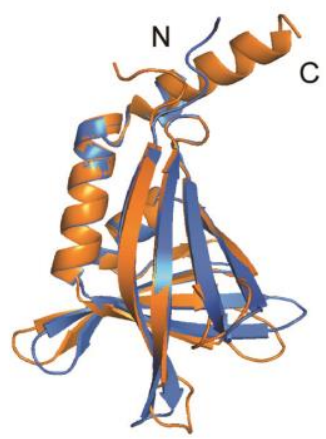

B
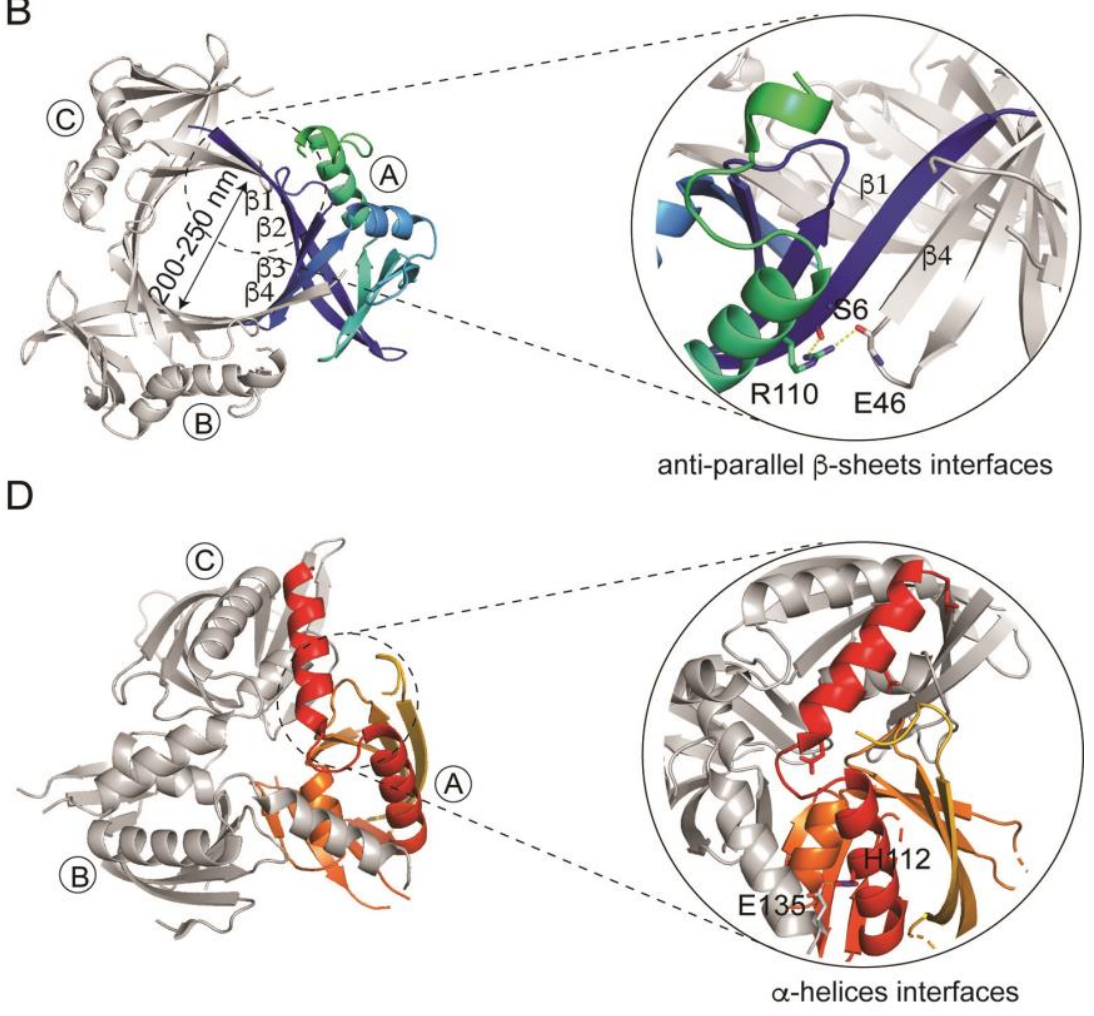

Figure 5

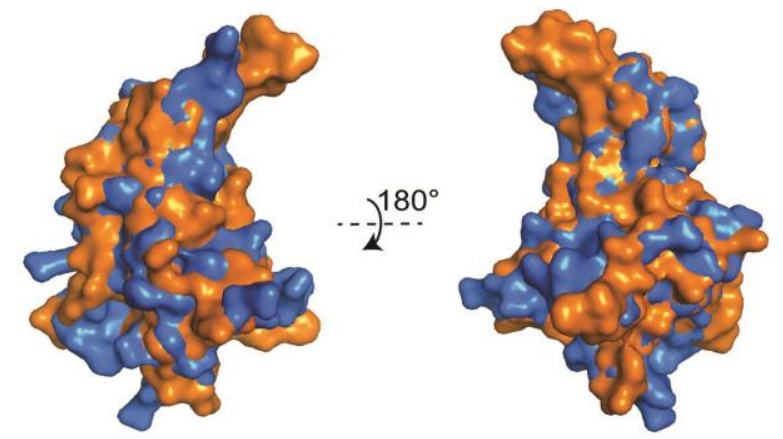


A

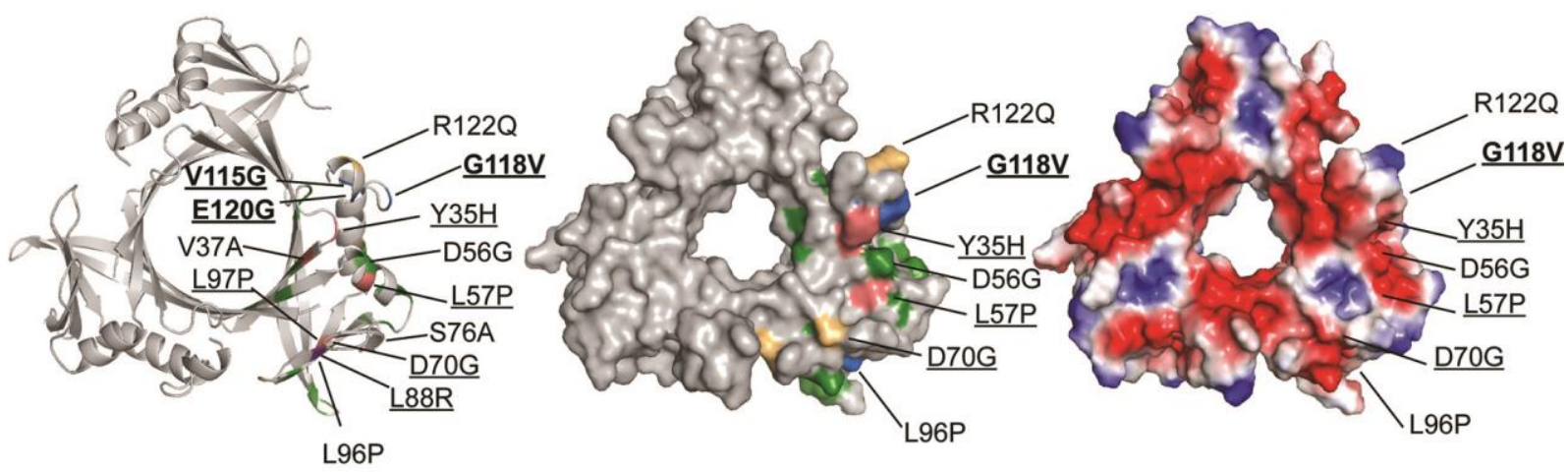

B

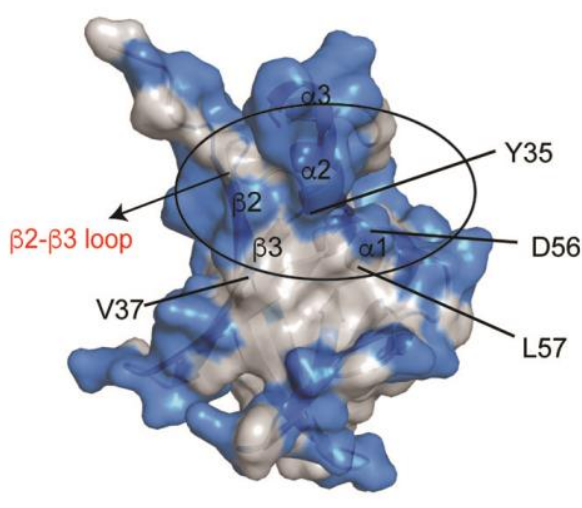

solvent exposed $\beta 6-\beta 7$ and $\beta 5-\alpha 1$ loop

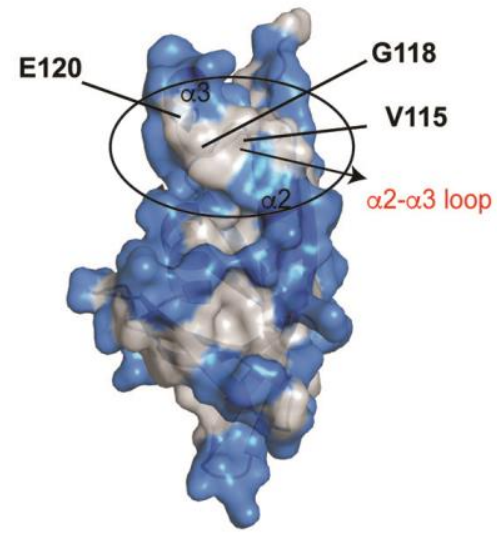

the interface of $\alpha 3$ with the rest of ScSsgB

C
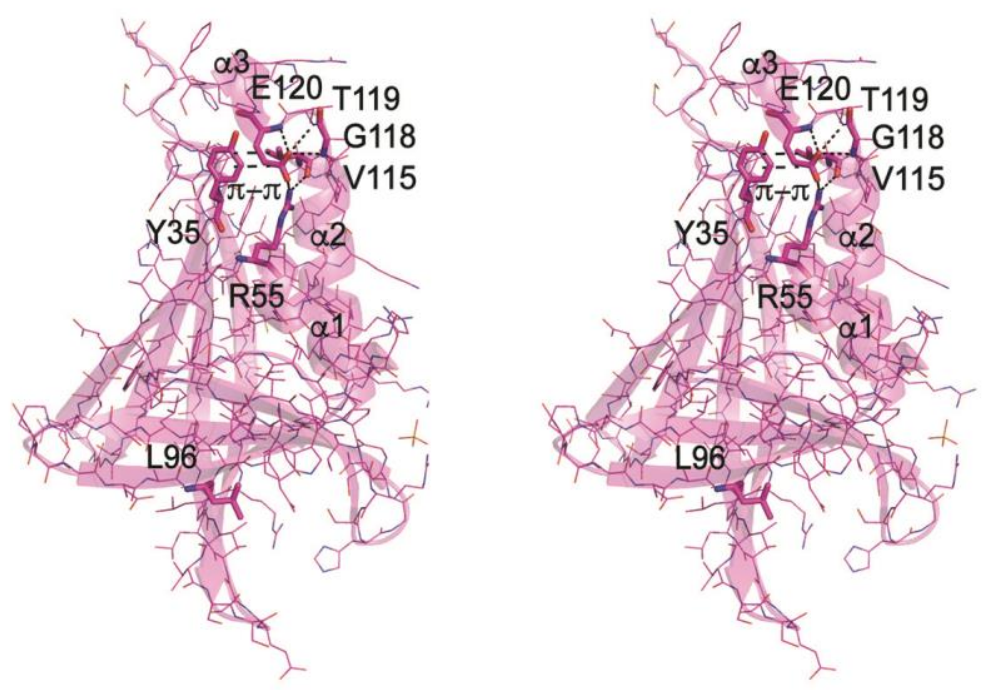

Figure 6 
A

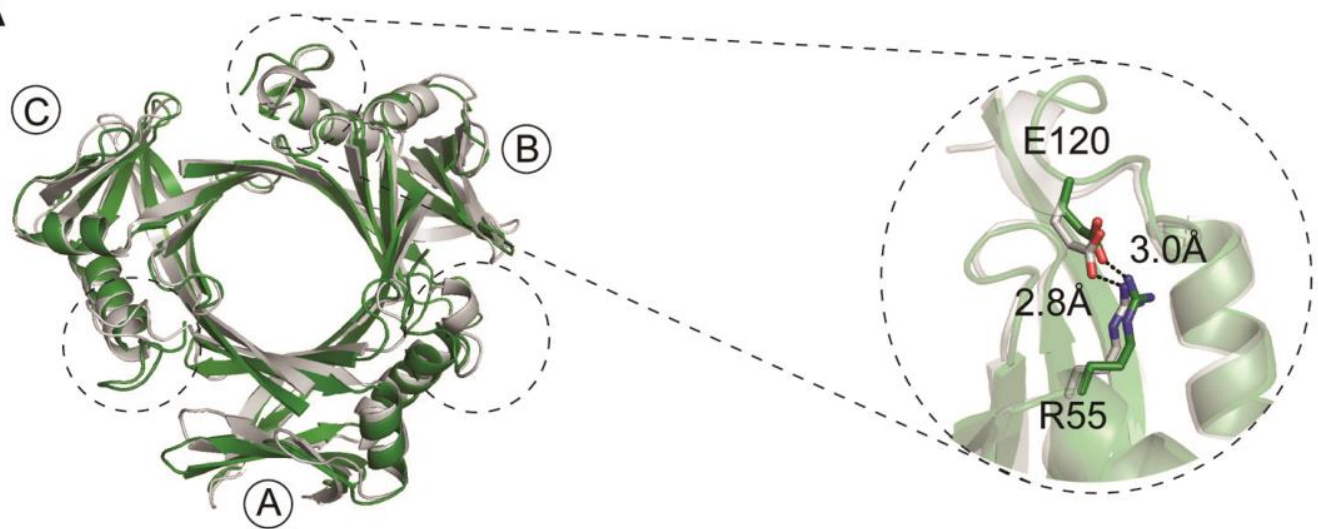

B

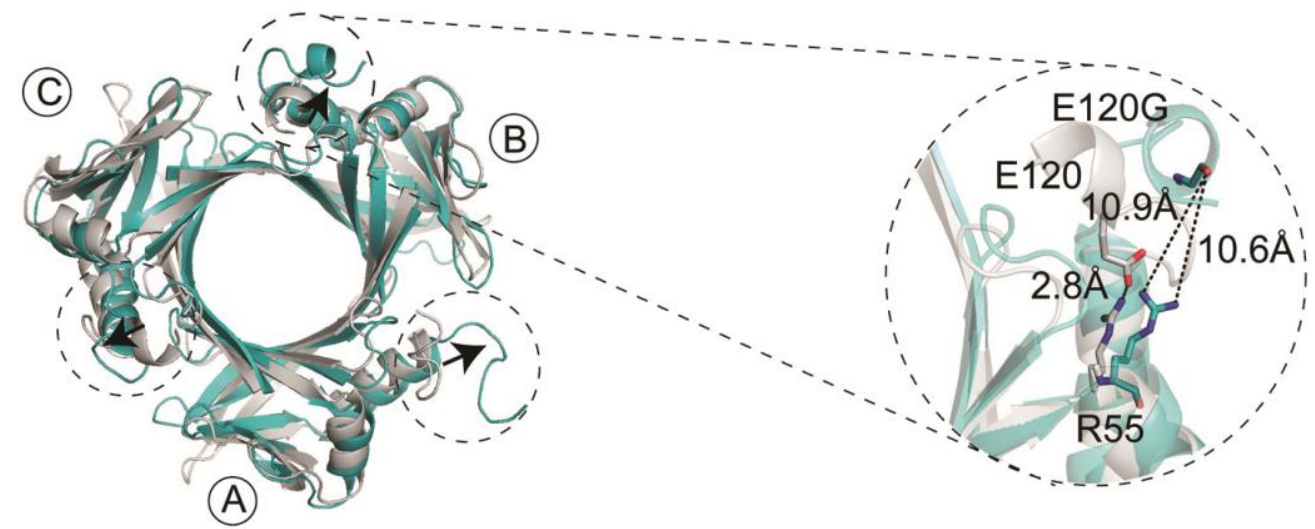

C

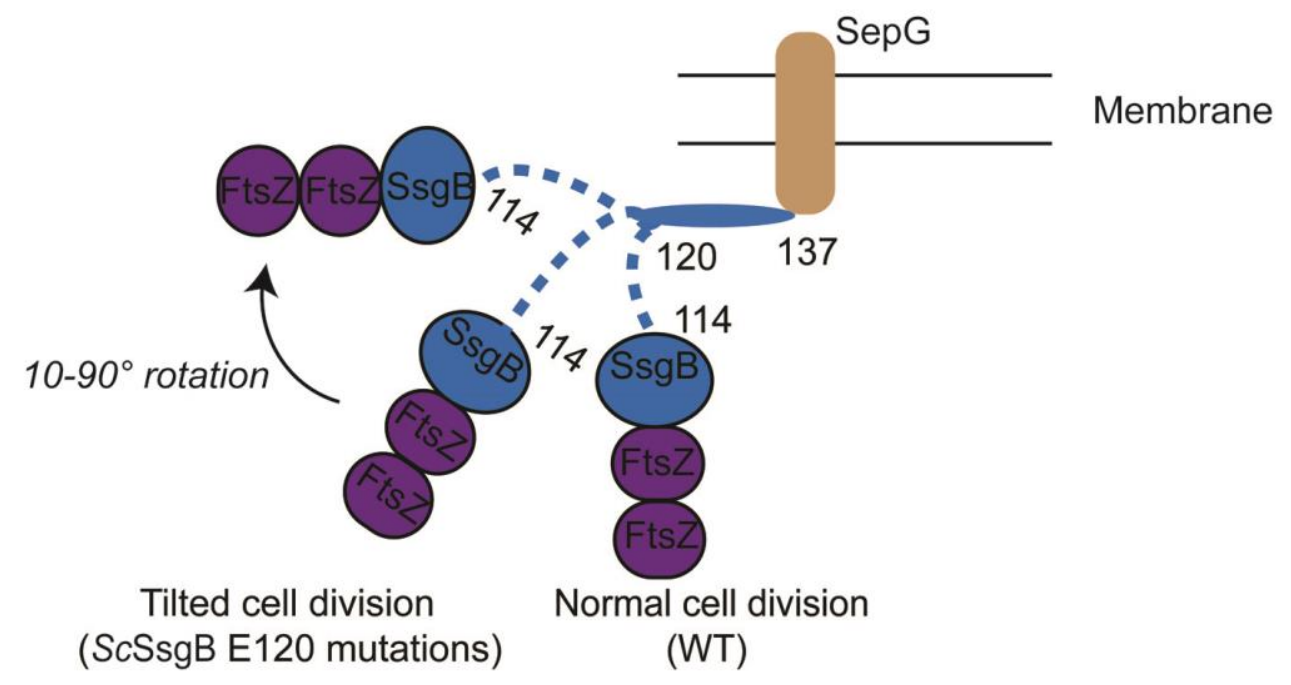

Figure 7 